\title{
A Design Algorithm using External Perturbation to Improve Iterative Feedback Tuning Convergence
}

Huusom, Jakob Kjøbsted; Hjalmarsson, Håkan; Poulsen, Niels Kjølstad; Jørgensen, Sten Bay

Published in:

Automatica

Link to article, DOI:

10.1016/j.automatica.2011.05.029

Publication date:

2011

Link back to DTU Orbit

Citation (APA):

Huusom, J. K., Hjalmarsson, H., Poulsen, N. K., \& Jørgensen, S. B. (2011). A Design Algorithm using External Perturbation to Improve Iterative Feedback Tuning Convergence. Automatica, 47(12), 2665-2670.

https://doi.org/10.1016/j.automatica.2011.05.029

\section{General rights}

Copyright and moral rights for the publications made accessible in the public portal are retained by the authors and/or other copyright owners and it is a condition of accessing publications that users recognise and abide by the legal requirements associated with these rights.

- Users may download and print one copy of any publication from the public portal for the purpose of private study or research.

- You may not further distribute the material or use it for any profit-making activity or commercial gain

- You may freely distribute the URL identifying the publication in the public portal 


\title{
A Design Algorithm using External Perturbation to Improve Iterative Feedback Tuning Convergence
}

\author{
Jakob K. Huusom $^{\text {a }}$, Håkan Hjalmarsson ${ }^{\text {b }}$, Niels K. Poulsen ${ }^{\text {c }}$, Sten B. Jørgensen ${ }^{\text {a }}$ \\ ${ }^{a}$ Department of Chemical and Biochemical Engineering, Technical University of Denmark, DK - 2800 Lyngby, Denmark \\ ${ }^{\mathrm{b}}$ Department of Signals, Sensors and Systems, Royal Institute of Technology, SE - 10044 Stockholm, Sweden \\ ${ }^{\mathrm{c}}$ Department of Informatics and Mathematical Modelling, Technical University of Denmark, DK - 2800 Lyngby, Denmark
}

\begin{abstract}
Iterative Feedback Tuning constitutes an attractive control loop tuning method for processes in the absence of process insight. It is a purely data driven approach for optimization of the loop performance. The standard formulation ensures an unbiased estimate of the loop performance cost function gradient, which is used in a search algorithm for minimizing the performance cost. A slow rate of convergence of the tuning method is often experienced when tuning for disturbance rejection. This is due to a poor signal to noise ratio in the process data. A method is proposed for increasing the data information content by introducing an optimal perturbation signal in the tuning algorithm. The theoretical analysis is supported by a simulation example where the proposed method is compared to an existing method for acceleration of the convergence by use of optimal prefilters.
\end{abstract}

Key words: Controller tuning, Direct tuning, Iterative schemes, Iterative Feedback Tuning

\section{Introduction}

Control design and tuning for disturbance rejection is one of the classical disciplines in control theory and control engineering science. Design of compensators for disturbance rejection is well documented $[1,4,2]$. Given a particular control design, the tuning of the control parameters can be conducted based on tuning rules or by minimization of some loop performance criterion. Given a model of the system, the set of optimal control parameters which minimize the performance cost can be evaluated. In absence of a sufficiently reliable model, the tuning can be performed based on data obtained from the loop, by a data driven optimization. Iterative Feedback Tuning is a method for optimizing control parameters using closed loop data which forms the basis for the modifications presented here. The basic algorithm was first presented in [14] and has since then been analyzed, extended and tested in a number of papers. For an extensive overview of the development of the method and references to applications, see $[6,12,15]$. Alternative data

Email addresses: jkh@kt.dtu.dk (Jakob K. Huusom), hakan.hjalmarsson@ee.kth.se (Håkan Hjalmarsson), nkp@imm.dtu.dk (Niels K. Poulsen), sbj@kt.dtu.dk (Sten B. Jørgensen). driven tuning algorithms are Correlation based Tuning $[18,19]$ and Virtual Reference Feedback Tuning $[5,20]$.

The performance criterion, $F_{N}\left(y_{t}, u_{t}\right)$, used in the controller tuning is a function of the output and the control action. Hence it is a function of the true system, the controller and external signals acting on the loop. We will use the set-up in Fig. 1 where $G$ is a causal scalar linear time-invariant system, $C$ is the controller, which also is assumed to be causal scalar linear time-invariant, and where $r_{t}$ is the reference signal and $v_{t}$ is the disturbance, respectively. Assuming, as we will, that the disturbance is stochastic implies that the performance cost is itself a random variable. However, as in, e.g., LQG-control, it is natural to minimize the expected cost

$$
F(\cdot) \triangleq \mathrm{E}\left[F_{N}(\cdot)\right]
$$

where $\mathrm{E}[\cdot]$ is the mathematical expectation over the random disturbances acting on the closed loop system. Notice that in the following, when expectation of $F(\cdot)$ is taken, the expectation refers not to the random disturbances acting on the system when assessing the closed loop performance. Instead it refers to the random variables that have affected the experimental data that has been used to design the controller for which the performance of $F(\cdot)$ is to be assessed. 


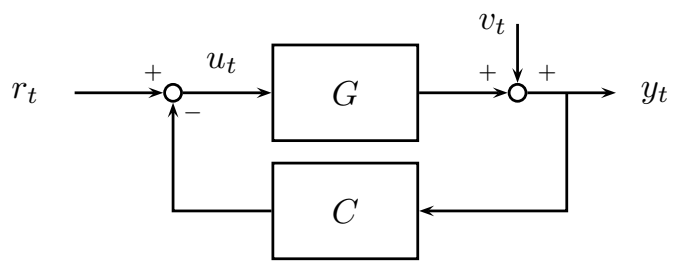

Fig. 1. A general feedback loop designed for disturbance rejection. The process, $G$, and the compensator in the feedback loop, $C$, is given as scalar linear transfer functions.

Our objective is to design a controller such that $F$ is minimized when $r_{t} \equiv 0$, i.e. we are interested in disturbance rejection. Adding a reference signal during the experimentation phase may however improve the quality of the obtained controller $C$. In Iterative Feedback Tuning, one tries to minimize $F$ with respect to the controller using noisy closed loop experiments. The accuracy of this design very much depends on the shape of the cost function $F$ one tries to minimize. Any change in the spectrum $\Phi_{r}$ of the reference signal, will affect the output spectrum $\Phi_{y}$ and the input spectrum $\Phi_{u}$. Hence the reference signal spectrum affects the minimum and the shape of the performance cost surface. By designing the spectrum of an external reference it is consequently possible to shape the performance cost function in order to improve the convergence properties of the search algorithm. However, one has to bear in mind that shaping the cost function will also influence the location of the minimum in the controller parameter space. Despite this unfortunate consequence, successful simulation studies are reported with respect to convergence using Iterative Feedback Tuning with external perturbation, when tuning for disturbance rejection [16].

\subsection{Formulating a design criterion}

Let $F(\boldsymbol{\rho}, \boldsymbol{\vartheta})$ denote the cost function that we are interested in minimizing, where $\boldsymbol{\rho}$ and $\boldsymbol{\vartheta}$ represent the free control parameters which are to be tuned and a set of parameters which characterize the reference signal spectrum, respectively. The objective is to find the optimal $\boldsymbol{\rho}$ for a given $\boldsymbol{\vartheta}=\boldsymbol{\vartheta}^{0}$, where $\boldsymbol{\vartheta}^{0}$ corresponds to $r_{t} \equiv 0$. We denote the optimum $\boldsymbol{\rho}$ by $\overline{\boldsymbol{\rho}}(\boldsymbol{\vartheta})$, indicating its dependence on $\boldsymbol{\vartheta}$. Since the system will be affected by noise it is only possible to obtain a minimizer, $\hat{\boldsymbol{\rho}}_{n}(\boldsymbol{\vartheta})$, with a certain accuracy; we use subscript $n$ to denote that $n$ iterations are performed in the tuning method. Hence Iterative Feedback Tuning will produce a solution with the following error

$$
\boldsymbol{\Sigma}_{n}(\boldsymbol{\vartheta}) \triangleq \mathrm{E}\left[\left(\hat{\boldsymbol{\rho}}_{n}(\boldsymbol{\vartheta})-\overline{\boldsymbol{\rho}}(\boldsymbol{\vartheta})\right)\left(\hat{\boldsymbol{\rho}}_{n}(\boldsymbol{\vartheta})-\overline{\boldsymbol{\rho}}(\boldsymbol{\vartheta})\right)^{T}\right]
$$

Using a continuity argument it may therefore be advantageous to optimize $\boldsymbol{\rho}$ for a $\boldsymbol{\vartheta} \neq \boldsymbol{\vartheta}^{0}$, i.e. it may be that the controller corresponding to $\boldsymbol{\vartheta}$ may result in a smaller expected cost for the desired excitation conditions (which correspond to $\boldsymbol{\vartheta}^{0}$ ) than the controller tuned with the desired operating conditions $\boldsymbol{\vartheta}^{0}$ i.e. $\mathrm{E}\left[F\left(\hat{\boldsymbol{\rho}}_{n}(\boldsymbol{\vartheta}), \boldsymbol{\vartheta}^{0}\right)\right]<$ $\mathrm{E}\left[F\left(\hat{\boldsymbol{\rho}}_{n}\left(\boldsymbol{\vartheta}^{0}\right), \boldsymbol{\vartheta}^{0}\right)\right]$ Our objective is to determine operating conditions $\boldsymbol{\vartheta}$ such that $\mathrm{E}\left[F\left(\hat{\boldsymbol{\rho}}_{n}(\boldsymbol{\vartheta}), \boldsymbol{\vartheta}^{0}\right)\right]$ is minimized. This is a difficult problem since $F\left(\hat{\boldsymbol{\rho}}_{n}(\boldsymbol{\vartheta}), \boldsymbol{\vartheta}^{0}\right)$ is a complicated and non-linear function of the random disturbances originating from the experiments on which $\hat{\boldsymbol{\rho}}_{n}(\boldsymbol{\vartheta})$ is based. This in turn means that the expectation with respect to these random variables is difficult to compute. Our approach to cope with this is to perform a local analysis, assuming $\boldsymbol{\vartheta}$ to be close to $\boldsymbol{\vartheta}^{0}$. Using Taylor expansion to second order near the optimum gives

$$
\begin{aligned}
& F\left(\hat{\boldsymbol{\rho}}_{n}(\boldsymbol{\vartheta}), \boldsymbol{\vartheta}^{0}\right) \approx F\left(\overline{\boldsymbol{\rho}}\left(\boldsymbol{\vartheta}^{0}\right), \boldsymbol{\vartheta}^{0}\right)+ \\
& \frac{1}{2} \operatorname{Tr}\left\{\frac{\partial^{2} F\left(\overline{\boldsymbol{\rho}}\left(\boldsymbol{\vartheta}^{0}\right), \boldsymbol{\vartheta}^{0}\right)}{\partial \boldsymbol{\rho}^{2}}\left(\hat{\boldsymbol{\rho}}_{n}(\boldsymbol{\vartheta})-\overline{\boldsymbol{\rho}}\left(\boldsymbol{\vartheta}^{0}\right)\right)\left(\hat{\boldsymbol{\rho}}_{n}(\boldsymbol{\vartheta})-\overline{\boldsymbol{\rho}}\left(\boldsymbol{\vartheta}^{0}\right)\right)^{T}\right\}
\end{aligned}
$$

By taking the expectation and rearranging using equation (2) it is seen that

$$
\begin{aligned}
& \mathrm{E}\left[F\left(\hat{\boldsymbol{\rho}}_{n}(\boldsymbol{\vartheta}), \boldsymbol{\vartheta}^{0}\right)\right]-F\left(\overline{\boldsymbol{\rho}}\left(\boldsymbol{\vartheta}^{0}\right), \boldsymbol{\vartheta}^{0}\right) \approx \\
& \frac{1}{2} \operatorname{Tr}\left\{\frac{\partial^{2} F\left(\overline{\boldsymbol{\rho}}\left(\boldsymbol{\vartheta}^{0}\right), \boldsymbol{\vartheta}^{0}\right)}{\partial \boldsymbol{\rho}^{2}}\left(\overline{\boldsymbol{\rho}}(\boldsymbol{\vartheta})-\overline{\boldsymbol{\rho}}\left(\boldsymbol{\vartheta}^{0}\right)\right)\left(\overline{\boldsymbol{\rho}}(\boldsymbol{\vartheta})-\overline{\boldsymbol{\rho}}\left(\boldsymbol{\vartheta}^{0}\right)\right)^{T}\right\} \\
& +\frac{1}{2} \operatorname{Tr}\left\{\frac{\partial^{2} F\left(\overline{\boldsymbol{\rho}}\left(\boldsymbol{\vartheta}^{0}\right), \boldsymbol{\vartheta}^{0}\right)}{\partial \boldsymbol{\rho}^{2}} \boldsymbol{\Sigma}_{n}(\boldsymbol{\vartheta})\right\} \triangleq \Delta F_{n}(\boldsymbol{\vartheta})
\end{aligned}
$$

Now, if the covariance, $\boldsymbol{\Sigma}_{n}(\boldsymbol{\vartheta})$, can be evaluated then $\Delta F_{n}(\boldsymbol{\vartheta})$ is a quantity that can be minimized with respect to $\vartheta$ in order to find the (approximately) optimal (reference) perturbation signal spectrum to be used in the experiments when tuning the controller parameters $\boldsymbol{\rho}$ using Iterative Feedback Tuning. The two terms in $\Delta F_{n}(\boldsymbol{\vartheta})$ can be interpreted as follows: The first term is the bias error due to that $\boldsymbol{\vartheta} \neq \boldsymbol{\vartheta}^{0}$ is used in the optimization whereas the second term is the variance error incurred on $F\left(\hat{\boldsymbol{\rho}}_{n}(\boldsymbol{\vartheta}), \boldsymbol{\vartheta}^{0}\right)$. The bias error will typically increase as $\boldsymbol{\vartheta}$ moves away from $\boldsymbol{\vartheta}^{0}$. As noted above, it may be possible to decrease the variance error if $\boldsymbol{\vartheta}$ is suitably chosen. The optimal perturbation choice $\boldsymbol{\vartheta}=\overline{\boldsymbol{\vartheta}}$ will balance these two terms. The aim of this study is to construct a systematic and formal algorithm for designing an optimal external perturbation signal for Iterative Feedback Tuning for the disturbance rejection problem based on (3).

The paper is organized as follows: Section 2 presents the basic Iterative Feedback Tuning algorithm for disturbance rejection and the error $\boldsymbol{\Sigma}_{n}(\boldsymbol{\vartheta})$ of the method derived in [11]. In Section 3 the effect of adding an external perturbation signal to the loop in the tuning method is analyzed. In Section 4, a formal design criterion for the perturbation spectrum in Perturbed Iterative Feedback Tuning is derived. Finally a simulation example serves to illustrate the advantages of introducing optimal external perturbation when tuning the loop for disturbance rejection. 


\section{Iterative Feedback Tuning for disturbance re- jection}

The Iterative Feedback Tuning algorithm for disturbance rejection is illustrated in the following [13]. The feedback loop in Fig. 1 depicts the signals and transfer functions which are used in the algorithm for tuning the parameters $\rho$ in $C$. The objective is minimization of the cost function:

$$
F\left(\boldsymbol{\rho}_{i}\right)=\frac{1}{2 N} \mathrm{E}\left[\sum_{t=1}^{N}\left(y_{t}\left(\boldsymbol{\rho}_{i}\right)-y_{t}^{d}\right)^{2}+\lambda\left(u_{t}\left(\boldsymbol{\rho}_{i}\right)\right)^{2}\right]
$$

where $N$ is the number of discrete time data points and $y^{d}=0$ is the desired output response for disturbance rejection. The sensitivity of the cost function with respect to the control parameters is

$$
\boldsymbol{J}\left(\boldsymbol{\rho}_{i}\right)=\frac{1}{N} \mathrm{E}\left[\sum_{t=1}^{N} y_{t}\left(\boldsymbol{\rho}_{i}\right) \frac{\partial y_{t}\left(\boldsymbol{\rho}_{i}\right)}{\partial \boldsymbol{\rho}}+\lambda u_{t}\left(\boldsymbol{\rho}_{i}\right) \frac{\partial u_{t}\left(\boldsymbol{\rho}_{i}\right)}{\partial \boldsymbol{\rho}}\right]
$$

The minimization is realized by iterating in the scheme

$$
\boldsymbol{\rho}_{i+1}=\boldsymbol{\rho}_{i}-\gamma_{i} \mathbf{R}_{i}^{-1} \boldsymbol{J}\left(\boldsymbol{\rho}_{i}\right)
$$

where $\mathbf{R}_{i}$ is a positive definite matrix typically chosen as the Hessian of the cost function with respect to the control parameters. If a model for the system is unknown, the gradients of the input and output and hence the cost function gradient cannot be evaluated analytically. In the traditional Iterative Feedback Tuning framework the minimization of the cost function, (4), is based on data from two successive experiments:

- Collect data $\left\{y_{t}^{1}\left(\boldsymbol{\rho}_{i}\right), u_{t}^{1}\left(\boldsymbol{\rho}_{i}\right)\right\}_{t=1, \ldots, N}$ where $r_{t}^{1}=0$

- Collect data $\left\{y_{t}^{2}\left(\boldsymbol{\rho}_{i}\right), u_{t}^{2}\left(\boldsymbol{\rho}_{i}\right)\right\}_{t=1, . ., N}$ where $r_{t}^{2}=-y_{t}^{1}$

This data is used to estimate the derivative of the cost function in (5) with $\left\{y_{t}, u_{t}\right\}=\left\{y_{t}^{1}, u_{t}^{1}\right\}$ and

$$
\begin{aligned}
& \frac{\widehat{\partial y_{t}}}{\partial \boldsymbol{\rho}} \triangleq \frac{\partial C\left(\boldsymbol{\rho}_{i}\right)}{\partial \boldsymbol{\rho}} y_{t}^{2} \\
& \frac{\partial u_{t}}{\partial \boldsymbol{\rho}} \triangleq \frac{\partial C\left(\boldsymbol{\rho}_{i}\right)}{\partial \boldsymbol{\rho}} u_{t}^{2}
\end{aligned}
$$

where (7) and (8), are the estimators for the gradients of the input and output. Given that the noise $v$ is a zero mean, weakly stationary random signal, the key contribution in Iterative Feedback Tuning, is that it supplies an unbiased estimate of the cost function gradient, without requiring a plant model estimate. The unbiased estimate of (5) consists of two terms: A term for the desired gradient, $S_{N}$, and a variance term, $E_{N}$. The latter term is due to the noise present in the second experiment [13]. A Gauss-Newton approximation of the Hessian to the performance cost function which is computed from these estimates was suggested in [14].

\subsection{Asymptotic accuracy of the tuning method}

The stochastic contribution in the gradient estimate will affect the asymptotic convergence rate of the tuning method. A quantitative analysis was performed by [10]. The result is as follows: With $n$ being the iteration number and $\bar{\rho}$ the optimal set of parameters, the sequence of random variables, $\sqrt{n}\left(\boldsymbol{\rho}_{n}-\overline{\boldsymbol{\rho}}\right)$, converge in distribution to a normally distributed random variable with zero mean and covariance matrix $\boldsymbol{\Sigma}$ according to

$$
\begin{aligned}
& \sqrt{n}\left(\boldsymbol{\rho}_{n}-\overline{\boldsymbol{\rho}}\right) \stackrel{D}{\rightarrow} \mathcal{N}(0, \boldsymbol{\Sigma}) \\
& \boldsymbol{\Sigma}=a^{2} \int_{0}^{\infty} e^{\boldsymbol{A} t} \boldsymbol{R}^{-1} \operatorname{Cov}[\widehat{\boldsymbol{J}(\overline{\boldsymbol{\rho}})}] \boldsymbol{R}^{-1} e^{\boldsymbol{A}^{T} t} d t
\end{aligned}
$$

The result in (9) is valid given the following conditions:

(1) The sequence $\boldsymbol{\rho}_{n}$ converges to a local isolated mini$\operatorname{mum} \overline{\boldsymbol{\rho}}$ of $F$

(2) $\boldsymbol{H}(\overline{\boldsymbol{\rho}})$ is the Hessian for $F(\boldsymbol{\rho})$ at $\overline{\boldsymbol{\rho}}$.

(3) The gain sequence $\left\{\gamma_{n}\right\}$ in (6) is given by $\gamma_{n}=a / n$, where $\mathrm{a}$ is a positive constant.

(4) There exists an index $\bar{n}$ and a matrix $\boldsymbol{R}$ such that $\boldsymbol{R}_{n}=\boldsymbol{R}$ for all $n>\bar{n}$.

(5) The matrix $\boldsymbol{A}=1 / 2 \boldsymbol{I}-a \boldsymbol{R}^{-1} \boldsymbol{H}(\overline{\boldsymbol{\rho}})$ is stable, i.e. the real parts of all the eigenvalues are negative.

(6) The covariance matrix $\operatorname{Cov}[\widehat{\boldsymbol{J}(\overline{\boldsymbol{\rho}})}]$ is positive definite.

The result in (9) means that asymptotically the distribution for the deviation between the $n$ 'th iterate of the controller parameter and the true optimum is known, and that the method converges to the true local minimizer of the performance cost function. In [11] it is shown that the covariance expression for the distribution simplifies if $\boldsymbol{H}(\overline{\boldsymbol{\rho}})$, i.e. the true Hessian, is used as the matrix $\boldsymbol{R}$ in (6). Hence for a Newton-Raphson optimization

$$
\boldsymbol{\Sigma}=\frac{a^{2}}{2 a-1} \boldsymbol{R}^{-1} \operatorname{Cov}[\widehat{\boldsymbol{J}(\overline{\boldsymbol{\rho}})}] \boldsymbol{R}^{-1}
$$

As a measure of the quality of the controller for a given iteration, $n$, in the tuning algorithm [11] suggests the difference between the expected value of the performance cost with $C\left(\boldsymbol{\rho}_{n}\right)$ in the loop minus the theoretical minimum value. This quantity is by definition a positive measure and were approximated in (3) by $\Delta F_{n}$, which will be referred to as the control quality index. It is seen that the covariance of the gradient estimate for the performance cost function influences both the asymptotic covariance of the distribution of $\Delta \overline{\boldsymbol{\rho}}_{n}$ and also the control quality index, through (3), given the parameters $\boldsymbol{\rho}_{n}$. A decomposition of this covariance expression gives

$$
\operatorname{Cov}[\widehat{\boldsymbol{J}(\boldsymbol{\rho})}]=\operatorname{Cov}\left[S_{N}(\boldsymbol{\rho})\right]+\operatorname{Cov}\left[E_{N}(\boldsymbol{\rho})\right]
$$


The limits $\lim _{N \rightarrow \infty} N \operatorname{Cov}\left[S_{N}(\boldsymbol{\rho})\right]$ and $\lim _{N \rightarrow \infty} N \operatorname{Cov}\left[E_{N}(\boldsymbol{\rho})\right]$ are presented in $[10]$.

The following section will illustrate how external perturbations can improve the control quality index and accelerate convergence of the tuning method. In [13] it is shown how a similar acceleration can be achieved by filtering the reference signal before the two gradient experiments, and subsequently filtering of the input/output data from these experiments with the inverse of the filters. Optimal design of the prefilters, $W_{i}$, have been investigated in $[9,11]$ where the asymptotic accuracy of the tuning method is improved by minimizing the covariance of the gradient estimate. The optimal prefilter is

$$
\begin{aligned}
\left|W_{i}^{o p t}\left(e^{j \omega}\right)\right|^{4} & =\beta\left|S\left(e^{j \omega}, \boldsymbol{\rho}_{i}\right)\right|^{2} \Phi_{v}(\omega)\left[1+\lambda\left|C\left(e^{j \omega}, \boldsymbol{\rho}_{i}\right)\right|^{2}\right]^{2} \\
\times & \operatorname{Tr}\left\{\boldsymbol{R}_{i}^{-1} \frac{\partial C\left(e^{j \omega}, \boldsymbol{\rho}_{i}\right)}{\partial \boldsymbol{\rho}} \frac{\partial C^{*}\left(e^{j \omega}, \boldsymbol{\rho}_{i}\right)}{\partial \boldsymbol{\rho}}\right\}
\end{aligned}
$$

where the constant $\beta$ is given by the design restriction and $S$ is the sensitivity transfer function for the loop.

\section{Introducing external perturbations in the tuning}

It is desired to improve the convergence and the asymptotic accuracy of the tuning method. To achieve this, the signal to noise ratio in data must be increased. An external perturbation signal will be used as reference in the first of the two experiments used in the tuning algorithm. The experiments are then defined as follows:

- Collect data $\left\{y_{t}^{1}\left(\boldsymbol{\rho}_{i}\right), u_{t}^{1}\left(\boldsymbol{\rho}_{i}\right)\right\}_{t=1, . ., N}$ where $r_{t}^{1}=r_{t}^{p}$ - Collect data $\left\{y_{t}^{2}\left(\boldsymbol{\rho}_{i}\right), u_{t}^{2}\left(\boldsymbol{\rho}_{i}\right)\right\}_{t=1, \ldots, N}$ where $r_{t}^{2}=-y_{t}^{1}$

where the external input $r_{t}^{p}$ is characterized by the spectrum $\Phi_{r^{p}}$. A discussion on using external perturbations in the Iterative Feedback Tuning algorithm and an introduction to Perturbed Iterative Feedback Tuning are given in [16]. Introducing the external perturbation signal will affect the estimates of the input and output gradients in (7) and (8) and the two terms $S_{N}\left(\boldsymbol{\rho}_{i}\right)$ and $E_{N}\left(\boldsymbol{\rho}_{i}\right)$ in the gradient estimate of the cost function. Assuming the unknown disturbance, $\left\{v_{t}\right\}$, and the known reference signal, $r_{t}^{p}$, to be Gaussian processes and let a known realization of $r_{t}^{p}$ be used for all iterations in the tuning, then the asymptotic covariance expressions for $S_{N}(\boldsymbol{\rho})$ and $E_{N}(\boldsymbol{\rho})$ exists and the expressions are derived in [15]. From these covariance expressions and the Gauss-Newton estimate of the Hessian from data, it is seen how external perturbation will affect the relevant functions in relation to the covariance of the cost function gradient estimate. The asymptotic expressions for $S_{N}$ and $E_{N}$ are affine functions in the following variables: $\Phi_{r p}^{2}, \Phi_{v}^{2}$ and $\Phi_{r p} \Phi_{v}$, hence so is the asymptotic covariance estimate of the performance cost. The Hessian estimate is an affine function in $\Phi_{r^{p}}$ and $\Phi_{v}$ only.

\subsection{A special design for minimum variance control}

It would be interesting to have a design of $r_{t}^{p}$ which would not change the dynamics in the response of $y$ or $u$ compared to the unperturbed case. This is in general not possible [15] but for $\lambda=0$, i.e. minimum variance control, it is. From the general feedback loop, Fig. 1, it is seen that the closed loop transfer functions to the output are

$$
y_{t}=G S\left(\boldsymbol{\rho}_{i}\right) r_{t}^{p}+S\left(\boldsymbol{\rho}_{i}\right) v_{t}
$$

If $r_{t}^{p}=(\sqrt{\alpha} / G) v_{t}$ would be realizable, the output in (13) will simplify to $y_{t}=(1+\sqrt{\alpha}) S\left(\boldsymbol{\rho}_{i}\right) v_{t}$ which is only a scaled expression of the output for the unperturbed case which multiplied with -1 will be used as the reference to the gradient experiment. This perturbation signal design will render the gradient estimate unbiased for $\lambda=0$.

\subsection{External perturbations versus prefiltering}

The use of external perturbations in the tuning algorithm accelerates the convergence by improving the noise to signal ratio in data used for estimation of the cost function gradient. The prefiltering approach does similarly accelerate the convergence by suppressing the influence of the noise in a certain frequency band. Hence the perturbations give an active interaction with the loop while the prefiltering uses a passive. A formal comparison between the methods is difficult since several parameterizations of the perturbation signal can be considered. For simplicity the two methods are compared for $\lambda=0$ and $r_{t}^{p}=(\sqrt{\alpha} / G) \bar{v}_{t}$ where $\Phi_{\bar{v}}=\Phi_{v}$, hence knowledge of the system and the noise spectrum needs to be assumed in order to apply any of the two methods. The spectra of the output gradient estimate for the two approaches are

$$
\begin{aligned}
\Phi_{y^{\prime}}^{F i l t} & =\frac{\partial C\left(\boldsymbol{\rho}_{i}\right)}{\partial \boldsymbol{\rho}}\left(|T|^{2}|S|^{2} \Phi_{v}+\left|W_{i}\right|^{-2}|S|^{2} \Phi_{v}\right) \\
\Phi_{y^{\prime}}^{P I F T} & =\frac{\partial C\left(\boldsymbol{\rho}_{i}\right)}{\partial \boldsymbol{\rho}}\left(|T|^{2}|S|^{2}(1+\alpha) \Phi_{v}+|S|^{2} \Phi_{v}\right)
\end{aligned}
$$

where $T$ is the complementary sensitivity function. The first term is the deterministic part of the gradient estimate and the second is the noise term. For a fair comparison the input power in $r_{t}^{2}$ to the gradient experiment must be equal, i.e. $\mathrm{E}\left(W_{i} S v_{t}\right)^{2}=(1+\alpha) \mathrm{E}\left(S v_{t}\right)^{2}$

$$
\alpha=\frac{\mathrm{E}\left(W_{i} S v_{t}\right)^{2}-\mathrm{E}\left(S v_{t}\right)^{2}}{\mathrm{E}\left(S v_{t}\right)^{2}}
$$

In order to quantify which method would perform best the ratio between the deterministic and the variance part of the estimate (7) is evaluated for each method i.e. the signal to noise ratio. The ratio between these, $\Xi_{P I F T / F i l t}$, are computed as

$$
\Xi_{P I F T / F i l t}=\frac{\int\left|W_{i}\right|^{2}|S|^{2} \Phi_{v} d \omega \int\left|W_{i}\right|^{-2}|S|^{2} \Phi_{v} d \omega}{\int|S|^{2} \Phi_{v} d \omega \int|S|^{2} \Phi_{v} d \omega}
$$


When this ratio is larger than one Perturbed Iterative Feedback Tuning will give better performance that Iterative Feedback Tuning with optimal prefilters. The Cauchy-Schwarz inequality gives that $\Xi_{P I F T / F i l t} \geq 1$ for any system and for all filters $W_{i}$ given the restriction imposed in this section.

$$
\begin{aligned}
\left(\int|S F|^{2} d \omega\right)^{2} & =\left(\int\left|S F W_{i}\right|\left|S F W_{i}^{-1}\right| d \omega\right)^{2} \\
& \leq \int\left|S F W_{i}\right|^{2} d \omega \int\left|S F W_{i}^{-1}\right|^{2} d \omega
\end{aligned}
$$

where $F$ is a spectral factorization of $\Phi_{v}$. The expression (17) is not easily evaluated due to the complexity of the optimal prefilter. It is however seen that for $\lambda=0$, the squared magnitude function of the optimal filter (12) can be approximated by $\left|W_{i}^{o p t}\right|^{2}=\left|S\left(\rho_{i}\right)\right| \sqrt{\Phi_{v}}$. In this expression, the trace of the matrix and the constraint variable $\beta$ has been combined and set equal to one. This is possible since $\alpha$ is adjusted according to the prefilter through equation (16). Inserting $\left|W_{i}^{o p t}\right|^{2}$ in (17) yields

$$
\Xi_{P I F T / F i l t}=\frac{\int|S|^{3} \Phi_{v}^{3 / 2} d \omega \int|S| \Phi_{v}^{1 / 2} d \omega}{\int|S|^{2} \Phi_{v} d \omega \int|S|^{2} \Phi_{v} d \omega}
$$

It is seen that the closed loop transfer function from the noise to the output $S H$ is appearing in all the integrals.

\section{A formal design criterion for the perturbation spectrum}

The previous section has shown that Perturbed Iterative Feedback Tuning can improve the convergence and decrease the necessary number of iterations when the objective is disturbance rejection. From Section 1.1 we have seen that $\Delta F_{n}(\vartheta)$ in Eq. (3), the control quality index, is a suitable design criterion where $\boldsymbol{\vartheta}^{0}$ corresponding to a zero reference signal. Equation (9) and condition 1-6 in Sec. 2.1, gives that $\boldsymbol{\Sigma}_{n}(\boldsymbol{\vartheta})$ can be expressed as $\boldsymbol{\Sigma}_{n}(\boldsymbol{\vartheta}) \approx 1 / n \boldsymbol{\Sigma}(\boldsymbol{\vartheta})$ where $n$ is the number of iterations to be performed, and

$$
\begin{aligned}
& \boldsymbol{\Sigma}(\boldsymbol{\vartheta})=a^{2} \int_{0}^{\infty} e^{\boldsymbol{A} t} \boldsymbol{R}^{-1} \operatorname{Cov}[\widehat{\boldsymbol{J}(\overline{\boldsymbol{\rho}}, \boldsymbol{\vartheta})}] \boldsymbol{R}^{-1} e^{\boldsymbol{A}^{T} t} d t \\
& \operatorname{Cov}[\widehat{\boldsymbol{J}(\boldsymbol{\rho}, \boldsymbol{\vartheta})}]=\operatorname{Cov}\left[S_{N}(\boldsymbol{\rho}, \boldsymbol{\vartheta})\right]+\operatorname{Cov}\left[E_{N}(\boldsymbol{\rho}, \boldsymbol{\vartheta})\right]
\end{aligned}
$$

where asymptotic (in the experiment length $N$ ) expressions for $\operatorname{Cov}\left[S_{N}(\boldsymbol{\rho}, \boldsymbol{\vartheta})\right]$ and $\operatorname{Cov}\left[E_{N}(\boldsymbol{\rho}), \boldsymbol{\vartheta}\right]$ are given in [15]. In case the gain direction $\mathbf{R}$ in the Iterative Feedback Tuning algorithm (6) is taken as $\partial^{2} F(\overline{\boldsymbol{\rho}}(\boldsymbol{\vartheta}), \boldsymbol{\vartheta}) / \partial \boldsymbol{\rho}^{2}$, the approximation (10) can be used for $\boldsymbol{\Sigma}_{n}(\boldsymbol{\vartheta})$. When full process knowledge is available all quantities in $\Delta F_{n}(\boldsymbol{\vartheta})$ can be computed and thus one can optimize this criterion in order to obtain a reference signal spectrum suitable for Iterative Feedback Tuning of the control loop for disturbance rejection. Since the design criterion is based on a Taylor expansion it is recommended to introduce a constraint on the reference signal power in the optimization. There are many possibilities for parameterizing the reference spectrum. In the following section a straightforward method is used, where the filter coefficients are used as design variables $\boldsymbol{\vartheta}$. It is also possible to use a linear parameterization of the spectrum itself [17]. The Hessian needed to calculate the control quality index, $\partial^{2} F\left(\overline{\boldsymbol{\rho}}\left(\boldsymbol{\vartheta}^{0}\right), \boldsymbol{\vartheta}^{0}\right) / \partial \boldsymbol{\rho}^{2}$, may have to be replaced by an approximation. The reason is that the Hessian evaluated with perturbations, $\partial^{2} F(\overline{\boldsymbol{\rho}}(\boldsymbol{\vartheta}), \boldsymbol{\vartheta}) / \partial \boldsymbol{\rho}^{2}$, is used when evaluating $\boldsymbol{\Sigma}_{n}(\boldsymbol{\vartheta})$. The following approximation is proposed

$$
g(\boldsymbol{\vartheta}) \triangleq \frac{F\left(\overline{\boldsymbol{\rho}}(\boldsymbol{\vartheta}), \boldsymbol{\vartheta}^{0}\right)}{F(\overline{\boldsymbol{\rho}}(\boldsymbol{\vartheta}), \boldsymbol{\vartheta})} \frac{\partial^{2} F(\overline{\boldsymbol{\rho}}(\boldsymbol{\vartheta}), \boldsymbol{\vartheta})}{\partial \boldsymbol{\rho}^{2}}
$$

This approximation is accurate when

$$
\left.\nu \triangleq \frac{d}{d \boldsymbol{\rho}} \frac{\partial^{2} F(\overline{\boldsymbol{\rho}}(\boldsymbol{\vartheta}), \boldsymbol{\vartheta})}{\partial \boldsymbol{\rho}^{2}}\right|_{\boldsymbol{\vartheta}=\boldsymbol{\vartheta}^{0}}
$$

is small. Note that $g(\boldsymbol{\vartheta})$ has a first order derivative at $\boldsymbol{\vartheta}=\boldsymbol{\vartheta}^{0}$ given by $g^{\prime}\left(\boldsymbol{\vartheta}^{0}\right)=\nu$.

As in general experimental design algorithms, the evaluation of the optimal solution relies on knowledge of the true system which is not available $[8,7,3]$. Therefore, practical use of the method will have to rely on an initial plant model. However, since the cost function appears to be smooth in many problems, e.g. the example in the following section [15], the accuracy of this model does not seem to be critical. In [16], were Perturbed Iterative Feedback Tuning has been introduced; a different criterion for the perturbation signal design was used. That design, inspired by system identification literature, is focused on achieving rich information content in data by shaping the Hessian of the cost function i.e. making it large in some sense. The criterion proposed in this paper is less general but tailored for improving the convergence properties of the Iterative Feedback Tuning method.

\section{An example}

A simulation study is performed in order to illustrate the ideas and advantages of introducing external perturbations in the Iterative Feedback Tuning method when tuning for disturbance rejection. For simplicity the control loop used is a discrete-time linear time-invariant transfer function model, and the controller has only two adjustable parameters. The random disturbance acting on the system is $e_{t} \in \mathcal{N}_{i i d}\left(0, \sigma^{2}\right)$ where $\sigma=1$. The nomenclature refers to the block diagram in Fig. 1 
where $v_{t}=H(q) e_{t}$.

$$
\begin{array}{ll}
\text { Plant model: } & G(q)=\frac{q^{-1}-0.5 q^{-2}}{1-0.3 q^{-1}-0.28 q^{-2}} \\
\text { Noise model: } & H(q)=\frac{1}{1+0.9 q^{-1}} \\
\text { Controller: } & C(q)=\rho_{1}+\rho_{2} q^{-1}
\end{array}
$$

This system was used in [11] to test the advantages of optimal prefilters in Iterative Feedback Tuning for disturbance rejection with $\lambda=0.6$ in the performance cost function. We have adopted this example to relate external perturbations to prefilters in Iterative Feedback Tuning. Since we thus far have no way of suggesting a good structure for the perturbation filter when $\lambda \neq 0$ we will choose a structure which is identical to the inverted system model times the noise model. This choice is motivated by Section 3.1 and we believe this model to be sufficiently simple in terms of number of free parameters and complex in terms of structure for this example. The initial values for the filter parameters are selected as the model parameters $\boldsymbol{\theta}$.

$$
r_{t}^{p}=G_{r^{p}}(q) H(q) e_{t}, \quad e_{t} \in \mathcal{N}_{i i d}\left(0, \sigma^{2}\right)
$$

where

$$
\begin{aligned}
& G_{r^{p}}(q)=\frac{1+\vartheta_{1} q^{-1}+\vartheta_{2} q^{-2}}{q^{-1}+\vartheta_{3} q^{-2}} \\
& \boldsymbol{\vartheta}_{0}=\boldsymbol{\theta}=\left[\begin{array}{lll}
-0.3 & -0.28 & -0.5
\end{array}\right]^{T}
\end{aligned}
$$

hence non causal filtering is required due to the forward time shift. In this filter design the parameter $\alpha$ which adjusts the gain will not be included in $\boldsymbol{\vartheta}$ as a free parameter. Hence variance of the perturbation signal will be determined by the remaining free parameters. The optimal set of filter parameters can be determined by the minimization of the control quality index, $\Delta F_{n}\left(\Phi_{r^{p}}\right)$ as an unconstrained problem. The optimal perturbation power will be a trade off between the displacement of the optimal control parameters due to perturbation, and the distance between the expected and optimal performance. When applying this filter for the perturbation signal the parameter $\alpha$ can be used to constraint the signal power. The optimal solution based on full process insight where computed as

$$
\boldsymbol{\vartheta}_{\text {opt }}=\left[\begin{array}{lll}
-14.48 & -15.29 & 0.5247
\end{array}\right]^{T}
$$

In the following, four series of 1000 Monte Carlo experiments are performed each containing $n=10$ iterations in the tuning. Initially the loop starts with the optimal set of control parameters for the unperturbed operation, $\rho=\left[\begin{array}{ll}-0.832 & 0.433\end{array}\right]$. First standard Iterative Feedback Tuning is used as a base case, then two series of Perturbed Iterative Feedback Tuning where a white noise signal and the optimized perturbation signal are used respectively. Finally the tuning is performed using the optimal prefilter. In the last three cases, the perturbation signal or the prefilter is constrained such that the reference signal to the gradient experiment is twice that of the standard Iterative Feedback Tuning method. The results of these four trials are shown in Fig. 2 and in Table 1. Please note that the abscissa in Fig. 2b is different from the other three plots. The results in Fig. 2 and Table 1 clearly illustrate the advantage of speeding up the convergence when tuning for disturbance rejection. The optimal set of perturbation filter parameters significantly reduces both the variance of final control parameters from the 1000 Monte Carlo experiments, and the displacement of the optimal control parameter solution for the perturbed problem. It is also clearly seen that the spectral properties of the perturbation signal plays an important role since applying a white noise signal gives a performance which is much worse than standard Iterative Feedback Tuning and introduces a clear bias. Tuning with the optimized prefilter or perturbation signal gives comparable results in this case where $\lambda=0.6$. The mean square error of the resulting controllers is smallest when the Perturbed Iterative Feedback Tuning is applied.

\section{Conclusions}

The convergence properties of the Perturbed Iterative Feedback Tuning algorithm for optimizing control parameters for disturbance rejection problems, have been investigated and compared to standard Iterative Feedback Tuning and tuning with optimal prefilters. A control quality index for Perturbed Iterative Feedback Tuning is proposed which can be evaluated based on asymptotic expressions for the covariance of the cost function gradient. It is shown that using a deterministic external perturbation signal in the tuning, will affect the control quality index. The magnitude of the improvement depends on the power and the frequency content of the perturbation signal. An algorithm based on process insight for minimizing the control quality index has been proposed. This algorithm is shown to be able to produce a perturbation signal which significantly improves the control quality index. Hence Perturbed Iterative Feedback Tuning performs better than classical Iterative Feedback Tuning when tuning for disturbance rejection. A direct comparison between the uses of perturbations versus prefilters to accelerate the convergence of the tuning is complicated. The prefiltering design is based on an explicit expression given $|S|^{2} \Phi_{v}$ while the optimal perturbation signal is a result of an optimization requiring information about the open loop plant dynamics. For the minimum variance control design it is shown that Perturbed Iterative Feedback Tuning gives better signal to noise ratio for the gradient estimates, than Iterative Feedback Tuning with optimal prefilters. 

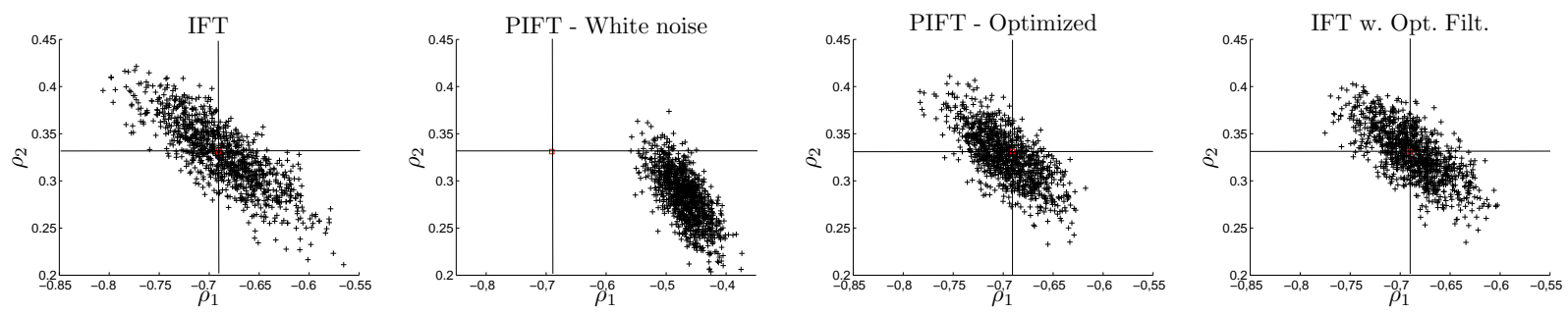

Fig. 2. The final control parameters from 1000 Monte Carlo experiments each with 10 iterations in standard IFT, PIFT with white noise and an optimized signal as perturbation signal and IFT with optimal prefilter. The optimal value for the control parameters for the unperturbed problem is marked with the straight lines.

\begin{tabular}{l|cc|ccc|c}
\hline Statistic & mean $\left(\boldsymbol{\rho}_{1}\right)$ & mean $\left(\boldsymbol{\rho}_{2}\right)$ & $\sigma_{\rho_{1}}^{2} \cdot 10^{3}$ & $\sigma_{\rho_{2}}^{2} \cdot 10^{3}$ & $\sigma_{\rho_{1}, \rho_{2}} \cdot 10^{3}$ & MSE·10 \\
\hline IFT & -0.686 & 0.328 & 1.67 & 1.30 & -1.20 & 2.99 \\
PIFT - white noise & -0.469 & 0.283 & 0.913 & 0.796 & -0.580 & 52.9 \\
PIFT - Optimized & -0.697 & 0.326 & 0.810 & 0.821 & -0.583 & 1.69 \\
IFT w. Opt. filt & -0.688 & 0.329 & 0.947 & 0.823 & -0.628 & 1.78 \\
\hline
\end{tabular}

Table 1

Mean, variance and the cross-covariance for the set of control parameters from 1000 Monte Carlo experiments with 10 iterations in the tuning method. Standard IFT, PIFT with white noise and an optimized perturbation signal and IFT with optimal prefilter for $\lambda=0.6$. The mean squared error of the parameters with respect to the optimal ones are presented in the last column

\section{References}

[1] Karl Johan Åström. Introduction to Stochastic Control Theory. Academic Press, 1970.

[2] Karl Johan Åström and Tore Hägglund. PID Controllers: Theory, Design and Tuning. Instrument Society of America, Research Triangle Park, North Carolina, 2ed edition, 1995.

[3] X Bombois, G Scorletti, M Gevers, R Hildebrand, and P van den Hof. Cheapest open-loop identification for control. In Proceedings of the 34rd IEEE Conference on Decision and Control, pages $382-387,2004$.

[4] G. E. P. Box and G. M. Jenkins. Time Series Analysis forecasting and control. Holden-Day, 1970.

[5] M. C. Campi, A. Lecchini, and S. M. Savaresi. Virtual reference feedback tuning: a direct method for the design of feedback controllers. Automatica, 28:1337 - 1346, 2002.

[6] Michel Gevers. A decade of progress in iterative process control design: from theory to practice. Journal of process control, 12(4):519-531, 2002.

[7] Michel Gevers and Lennard Ljung. Optimal experiment design with respect to the intended model application. Automatica, 22(5):543-554, 1986.

[8] Graham C. Goodwin and Robert L. Payne. Dynamic System Identification: Experiment Design and Data Analysis, volume 136 of Mathematics in science and engineering. Academic Press, 1977.

[9] R. Hildebrand, A. Lecchini, G. Solari, and M. Gevers. Prefiltering in iterative feedback tuning: Optimization of the prefilter for accuracy. IEEE Transactions on Automatic Control, 49(10):1801 - 1805, 2004.

[10] R. Hildebrand, A. Lecchini, G. Solari, and M. Gevers. Asymptotic accuracy of iterative feedback tuning. IEEE Transactions on Automatic Control, 50(8):1182 - 1185, 2005.

[11] R. Hildebrand, A. Lecchini, G. Solari, and M. Gevers. Optimal prefiltering in iterative feedback tuning. IEEE
Transactions on Automatic Control, 50(8):1196 - 1200, 2005.

[12] Håkan Hjalmarsson. Iterative feedback tuning - an overview. International journal of adaptive control and signal processing, 16:373-395, 2002.

[13] Håkan Hjalmarsson, Michel Gevers, Svante Gunnarsson, and Olivier Lequin. Iterative feedback tuning: Theory and applications. IEEE Control Systems Magazine, 18(4):26-41, 1998.

[14] Håkan Hjalmarsson, Svante Gunnarsson, and Michel Gevers. A convergent iterative restricted complexity control design scheme. In Proceedings of the 33rd IEEE Conference on Decision and Control, volume 2, pages 1735 - 1740, 1994.

[15] Jakob Kjøbsted Huusom. Identification for Control Developments in the Iterative Feedback Tuning Framework. $\mathrm{PhD}$ thesis, Technical University of Denmark, 2008. Url: http://orbit.dtu.dk/app.

[16] Jakob Kjøbsted Huusom, Niels Kjølstad Poulsen, and Sten Bay Jørgensen. Improving convergence of iterative feedback tuning. Journal of Process Control., 19(4):570-578, 2009.

[17] H. Jansson and H. Hjalmarsson. Input design via LMIs admitting frequency-wise model specifications in confidence regions. IEEE Transactions on Automatic Control, 50(10):1534-1549, 2005.

[18] A. Karimi, L. Mišković, and D. Bonvin. Iterative correlationbased controller tuning with application to a magnetic suspension system. Control Engineering Practice, 11(9):1069 $-1078,2003$.

[19] A. Karimi, L. Mišković, and D. Bonvin. Iterative correlationbased controller tuning. Int. journal of adaptive control and signal processing, 18(8):645 - 664, 2004.

[20] A. Lecchini, M. C. Campi, and S.M. Savaresi. Virtual reference feedback tuning for two degree of freedom controllers. International Journal of Adaptive Control and Signal Processing, 16:355-371, 2002. 